in vivo $34: 1797-1803(2020)$

doi:10.21873/invivo.11974

\title{
Ultrasonographic Follow-up of the Multistep Protocol for Prostate Cancer Induction in Wistar Rats
}

\author{
ANA I. FAUSTINO-ROCHA ${ }^{1,2}$, FERNANDA SEIXAS $^{3,4}$, RITA FERREIRA $^{5}$, JESSICA SILVA ${ }^{3}$, \\ MARIA J. PIRES ${ }^{2,3}$, MARGARIDA FARDILHA $^{6}$, MÁRIO GINJA $^{2,3^{*}}$ and PAULA A. OLIVEIRA ${ }^{2,3 *}$ \\ ${ }^{1}$ Faculty of Veterinary Medicine, Lusophone University of Humanities and Technologies, Lisbon, Portugal; \\ ${ }^{2}$ Center for the Research and Technology of Agro-Environmental and Biological Sciences (CITAB), Vila Real, Portugal; \\ ${ }^{3}$ Department of Veterinary Sciences, University of Trás-os-Montes and Alto Douro (UTAD), Vila Real, Portugal; \\ ${ }^{4}$ Animal and Veterinary Research Center (CECAV), Vila Real, Portugal; \\ ${ }^{5}$ Associated Laboratory for Green Chemistry of the Network of Chemistry and Technology (LAQV-REQUIMTE), \\ Department of Chemistry, University of Aveiro, Aveiro, Portugal; \\ ${ }^{6}$ Department of Medical Sciences, University of Aveiro, Aveiro, Portugal
}

\begin{abstract}
Aim: This work intended to improve the knowledge of the rat model of prostate cancer $(\mathrm{PCa})$ by ultrasonographic monitoring. Materials and Methods: Male Wistar rats were divided into control $(n=8)$ and $P C a(n=14)$ groups. PCa development was induced in the PCa group through the sequential administration of the anti-androgenic drug flutamide, testosterone propionate and the carcinogenic $N$-methyl-N-nitrosourea. The prostate was evaluated by ultrasonography at five timepoints along 49 weeks of the experimental protocol. Ventral prostate lobes were observed in all ultrasonographic examinations. Results: The ventral prostate area of the control group increased gradually between the first and the last ultrasonographic examination. The ventral prostate area of PCa groups decreased due to flutamide administration and increased after androgen and carcinogen administration. The area of the dorsal prostate lobe increased between the fourth and the fifth ultrasonographic examination. In the last ultrasonographic examination, hypoechoic and anechoic lesions were observed in the PCa group. Conclusion: To our knowledge, this is the first study presenting a follow-up of rat prostatic dimensions by ultrasonography. Ultrasonography is a feasible approach for prostate cancer monitoring in experimental models.
\end{abstract}

This article is freely accessible online.

*These Authors contributed equally to this work.

Correspondence to: Ana Faustino-Rocha, Campo Grande 376, 1749024 Lisbon, Portugal. Tel: +351 217515500, Fax: +351 217577006, e-mail: anafaustino.faustino@sapo.pt

Key Words: Carcinogenesis, prostate, rodent, ultrasonography.
The prostate is typically described as the largest accessory gland of the male reproductive tract (1). Together with other accessory glands of the reproductive tract, such as seminal vesicles and bulbourethral glands, the prostate is responsible for the production of an alkaline fluid that forms part of the seminal fluid (2).

After 40 years of age, in humans, the prostate is commonly affected by several pathologies, including benign prostate hyperplasia and cancer. Prostate cancer $(\mathrm{PCa})$ is one the most frequent types of cancer found in the male population (3). In 2018, PCa affected approximately 1.28 million men around the world and killed 358,989 of them (4). PCa development is associated with several risk factors, including older age, Black race/ethnicity, a family history of disease, an increased body mass index and obesity $(5,6)$. Although several therapeutic approaches are available to treat this disease, the number of PCa deaths is continuously increasing. The use of animal models to study PCa biopathology, prevention and treatment is of paramount importance. Although several species are available as models to study this disease, the rat is the most frequently used (79). Despite anatomical differences, prostate carcinogenesis in rats and men is controlled by similar molecular mechanisms, making the rat a valuable model for the study of human prostatic diseases, including cancer (7). Given the animal's size, it is not easy to assess the effectiveness of the protocol for cancer induction implemented in the laboratory. In most cases, the effectiveness, or not, of the protocol is only apparent after animals are sacrificed. Nevertheless, in certain experimental procedures, namely in those intended for studying potential therapeutic strategies, it is important to know tumor development in temporal terms. Thus, using a non-invasive/relatively cheap and accessible imaging tool, ultrasonography, we intended to perform, for the first time 
Table I. Animal body weight at sacrifice, and prostate absolute weight and relative weight (mean \pm S.E.).

\begin{tabular}{lccc}
\hline Group & $\begin{array}{c}\text { Body } \\
\text { weight }(\mathrm{g})\end{array}$ & $\begin{array}{c}\text { Prostate } \\
\text { weight }(\mathrm{g})\end{array}$ & $\begin{array}{c}\text { Relative prostate } \\
\text { weight }\end{array}$ \\
\hline Control (n=8) & $553.52 \pm 9.84$ & $1.11 \pm 0.07$ & $0.0020 \pm 0.0001$ \\
PCa (n=14) & $494.59 \pm 9.46^{\mathrm{a}}$ & $1.99 \pm 0.05^{\mathrm{a}}$ & $0.0041 \pm 0.0002^{\mathrm{a}}$ \\
\hline
\end{tabular}

aStatistically significantly different from the control group $(p<0.05)$.

as far as we are aware, follow-up of the multistep protocol for PCa in male Wistar rats in order to better characterize this animal model.

\section{Materials and Methods}

Animals. Twenty-two male Wistar Unilever rats (Rattus norvegicus) of 4 weeks of age were purchased from Charles River Laboratories (Charles River Laboratoire France, Écully, France). Animals were maintained under controlled conditions of temperature $\left(23 \pm 2^{\circ} \mathrm{C}\right)$, humidity $(50 \pm 10 \%)$, air system filtration (10-20 ventilations/hour) and light/dark cycle $(12 \mathrm{~h} / 12 \mathrm{~h})$. They had free access to water and a standard laboratory diet (Mucedola 4RF21 ${ }^{\circledR}$; Mucedola S.R.L., Settimo Milanese, Milan, Italy). Cages were cleaned, and water was changed weekly. All procedures were performed in accordance with the European Directive 2010/63/EU on the protection of animals used for scientific purposes. The Portuguese Ethics Committee for Animal Experimentation approved all experiments and procedures carried out on the animals (Direcção Geral Alimentação e Veterinária, approval no. 021326).

PCa induction. After a quarantine and an acclimatization period to laboratory conditions of 4 weeks, the animals were randomly divided into two experimental groups: Control group $(n=8)$ and $\mathrm{PCa}$ group $(n=14)$. PCa development was chemically and hormonally induced in the PCa group. At 12 weeks of age, animals from the $\mathrm{PCa}$ group received a subcutaneous administration of the antiandrogenic drug flutamide $(50 \mathrm{mg} / \mathrm{kg}$ of body weight; TCI Chemicals, Portland, OR, USA) for 21 consecutive days. Twentyfour hours after the last flutamide administration, testosterone propionate (TCI Chemicals) was dissolved in corn oil $(100 \mathrm{mg} / \mathrm{kg}$ of body weight) and subcutaneously administered to the animals. Forty-eight hours later, they were intraperitoneally injected with the carcinogenic agent $N$-methyl- $N$-nitrosourea (MNU; $(30 \mathrm{mg} / \mathrm{kg}$ of body weight, Isopac ${ }^{\circledR}$; Sigma Chemical Co., Madrid, Spain). After 2 weeks, silastic tubes were filled with crystalline testosterone (Sigma Chemical Co.) and subcutaneously implanted in the interscapular region of animals previously anesthetized with ketamine $\left(75 \mathrm{mg} / \mathrm{kg}\right.$, Imalgene ${ }^{\circledR} 1000$; Merial S.A.S., Lyon, France) and xylazine $\left(10 \mathrm{mg} / \mathrm{kg}\right.$, Rompun ${ }^{\circledR} 2 \%$; Bayer Healthcare S.A., Kiel, Germany). Animals were observed daily until the end of the experiment at 61 weeks of age to check their health status.

Prostate examination. The ultrasonographic examination of the rat prostate of both groups was performed in five time-points of the experimental protocol at 11,15, 21, 32 and 61 weeks of age. The ultrasonographic examinations were performed in awake animals,
Table II. Measurements of the radius of the major axis ( $r 1)$ and minor axis $(r 2)$ of the ventral prostate in the control and prostate cancer $(\mathrm{PCa})$ groups throughout the study (mean \pm S.E.).

\begin{tabular}{lccccc}
\hline \multirow{2}{*}{$\begin{array}{l}\text { Weeks } \\
\text { of age }\end{array}$} & \multicolumn{2}{c}{ Control $(\mathrm{n}=8)$} & & \multicolumn{2}{c}{ PCa $(\mathrm{n}=14)$} \\
\cline { 2 - 3 } \cline { 6 - 7 } & $\mathrm{r} 1(\mathrm{~cm})$ & $\mathrm{r} 2(\mathrm{~cm})$ & & $\mathrm{r} 1(\mathrm{~cm})$ & $\mathrm{r} 2(\mathrm{~cm})$ \\
\hline 11 & $0.76 \pm 0.03^{\mathrm{a}, \mathrm{b}}$ & $0.27 \pm 0.00$ & & $0.76 \pm 0.03^{\mathrm{b}, \mathrm{c}}$ & $0.30 \pm 0.01$ \\
15 & $0.69 \pm 0.05^{\mathrm{a}, \mathrm{b}}$ & $0.32 \pm 0.02$ & & $0.59 \pm 0.02^{\mathrm{b}, \mathrm{c}}$ & $0.26 \pm 0.01$ \\
21 & $0.92 \pm 0.03^{\mathrm{b}}$ & $0.33 \pm 0.01$ & & $1.05 \pm 0.04^{\mathrm{b}}$ & $0.39 \pm 0.01$ \\
32 & $0.82 \pm 0.07^{\mathrm{b}}$ & $0.31 \pm 0.03$ & & $1.11 \pm 0.08^{\mathrm{b}}$ & $0.38 \pm 0.02$ \\
61 & $1.04 \pm 0.08^{\mathrm{b}}$ & $0.31 \pm 0.08$ & & $1.05 \pm 0.05^{\mathrm{b}}$ & $0.35 \pm 0.02$
\end{tabular}

Statistically significantly different: afrom $\mathrm{r} 1$ at week 61 in the same group; bfrom $\mathrm{r} 2$ in the same group; ' from r1 at 21, 32 and 61 weeks in the same group $(p<0.05)$. No other statistically significant differences were found within nor between groups.

except for the last examination that coincided with the animals' sacrifice. The animals were restrained by a researcher and placed in the supine position. The skin of the inguinal region was shaved using a machine clipper (AESCULAP ${ }^{\circledR}$ GT420 Isis; Aesculap Inc, Center Valley, PA, USA). A real-time scanner $\left(\operatorname{Logic} \mathrm{P}^{\circledR}{ }^{\circledR}\right.$; General Electric Healthcare, Milwaukee, WI, USA) and a $12 \mathrm{MHz}$ linear transducer were used. Aquasonic acoustic gel (Parker Laboratories Inc., Fairfield, NJ, USA) was applied and a complete transverse scan using B mode was performed from the cranial to the caudal region of the prostate. The examinations were recorded and images with the greatest prostate dimensions were selected. Considering that the prostate lobes were elongated, having a major axis (the largest diameter) and a minor axis (the shortest diameter), they seemed to take on an ellipsoidal geometry. In this way, the largest (r1) and the smallest radius ( $\mathrm{r} 2$ ) of the dorsal and ventral prostate lobes were measured using the integral calipers of the ultrasound apparatus (the cursors were set at the borders of the prostate), and the dorsal and ventral prostate area was calculated using the ellipsoid formula: Area $=\pi \times r 1 \times r 2$.

The area of the ventral prostate of each animal was derived from the sum of the area of the left and right ventral lobes. At the last ultrasonographic examination (61 weeks of age), a contrast study of the prostate was performed to evaluate the vascularization of the prostate and prostatic lesions. System settings were optimized for the contrast-enhanced study with a mechanical index of 0.09 , and the gain compensation was adjusted for each animal/prostate. The position of the transducer was maintained during the examination. The sulfur hexafluoride microbubble contrast agent SonoVue (Bracco, Milan, Italy) was reconstituted by adding $5 \mathrm{ml}$ of a $0.9 \%$ saline solution. SonoVue was injected as a bolus $(0.1 \mathrm{ml})$ through a tail vein catheter, followed by a $1 \mathrm{ml}$ saline flush. The injection technique was carefully performed by the same researcher to avoid personal variations and produce acceptable and reproducible results. The perfusion process and dynamic enhancement of each prostate were observed in real time and continuously recorded in the ultrasound apparatus immediately after intravenous injection of the contrast agent.

Animal sacrifice and necropsy. Prior to sacrifice, animal body weight was measured using a top-loading scale (KERN ${ }^{\circledR}$ PLT 62002A; Dias de Sousa SA, Alcochete, Portugal). At 61 weeks of age, 
the animals were sacrificed by intraperitoneal administration of ketamine $\left(75 \mathrm{mg} / \mathrm{kg}\right.$, IMALGENE ${ }^{\circledR} 1000$; Merial S.A.S) and xylazine (10 mg/kg, Rompun ${ }^{\circledR} 2 \%$; Bayer Healthcare S.A.), followed by exsanguination by cardiac puncture as indicated by the Federation for Laboratory Animal Science Associations (10). Rats were necropsied and all relevant gross lesions were described. The prostate gland was removed, weighed and fixed in buffered formalin for at least 12 hours. For tissue preparation of prostate glands, two sagittal slices of the ventral prostate, three sagittal samples of the dorsolateral prostate including the urethra, and two longitudinal slices from each side of the anterior prostate and from seminal vesicles were embedded in paraffin.

Histological analysis. Each formalin-fixed prostate gland was processed for light microscopy by routine histological technique. Paraffin $3 \mu \mathrm{m}$-thick sections were stained with hematoxylin and eosin, and lesions were classified blindly to treatment group according to Bosland (11). The severity of proliferative/atrophic and inflammatory lesions was scored subjectively using four grades based on the extension of affected prostate tissue, as follows: Minimal lesion: occurring in less than $10 \%$ of prostate tissue; mild lesion: in 10-39\%; moderate lesion: in $40-50 \%$; and severe lesion: more than $50 \%$ of the prostate affected.

Statistical analysis. Data were statistically analyzed using SPSS ${ }^{\circledR}$ version 25 (IBM, Armonk, NY, USA). Continuous data were compared between groups using Student's $t$-test. Histological results were analyzed using chi-squared test. Continuous data are expressed as the mean \pm standard error (S.E.). Differences with $p$-values lower than 0.05 were considered statistically significant.

\section{Results}

Data availability statement. The data that support the findings of this study are available from the corresponding Author upon reasonable request.

Animals and prostate weight. All animals from both experimental groups exhibited a normal behavior and health status during the experimental protocol. At the end of the experiment, the body weight was lower in the PCa group when compared with the control group $(p<0.05)$. Prostate weight and relative prostate weight were higher in the $\mathrm{PCa}$ group than in the control group $(p<0.05)$ (Table I).

Prostate ultrasonographic examination. The prostate was easily evaluated by ultrasonography in awake animals from $\mathrm{PCa}$ and control groups. The ultrasonographic evaluation was performed at five timepoints, namely at 11, 15, 21, 32 and 61 weeks of age. Both ventral prostate lobes were observed in all ultrasonographic examinations. The dorsal prostate lobe was only visible in the last two ultrasonographic examinations for both groups .

The values of $\mathrm{r} 1$ for the ventral and dorsal prostate lodes were higher when compared with $\mathrm{r} 2$ in all ultrasonographic evaluations for both control and PCa groups $(p<0.05)$. For the control group, $\mathrm{r} 1$ increased between the first and the last
Table III. Measurements of the radius of the major axis ( $r 1)$ and minor axis ( $r 2$ ) of the dorsal prostate in the control and prostate cancer (PCa) groups throughout the study (mean \pm S.E.).

\begin{tabular}{lccccc}
\hline \multirow{2}{*}{$\begin{array}{l}\text { Weeks } \\
\text { of age }\end{array}$} & \multicolumn{2}{c}{ Control $(\mathrm{n}=8)$} & & \multicolumn{2}{c}{ PCa $(\mathrm{n}=14)$} \\
\cline { 2 - 3 } \cline { 5 - 6 } & $\mathrm{r} 1(\mathrm{~cm})$ & $\mathrm{r} 2(\mathrm{~cm})$ & & $\mathrm{r} 1(\mathrm{~cm})$ & $\mathrm{r} 2(\mathrm{~cm})$ \\
\hline 32 & $1.07 \pm 0.17^{\mathrm{a}}$ & $0.29 \pm 0.02$ & & $1.24 \pm 0.07^{\mathrm{a}}$ & $0.49 \pm 0.03$ \\
61 & $1.36 \pm 0.15^{\mathrm{a}}$ & $0.44 \pm 0.04$ & & $1.15 \pm 0.16^{\mathrm{a}}$ & $0.65 \pm 0.03$ \\
\hline
\end{tabular}

aStatistically significantly different from $\mathrm{r} 2(p<0.05)$. No other statistically significant differences were found within nor between groups.

ultrasonographic evaluation $(p<0.05)$. For the PCa group, $\mathrm{r} 1$ decreased between the first and the second examination, increased in the third and fourth, decreasing again at the last examination. In the last three ultrasonographic evaluations, r1 was statistically significantly higher than in the first and second examinations $(p<0.05)$. Statistically significant differences were not observed between $\mathrm{PCa}$ and control groups $(p>0.05)$ (Tables II and III).

The area of the ventral prostate of animals from the control group gradually increased from the first to the last ultrasonographic examination. The ventral prostate area of animals from the PCa group decreased between the first and the second ultrasonographic examination. An increase of the prostate area of the PCa group was observed between the second and the fourth ultrasonographic examination $(p<0.05)$. The ventral prostate area decreased between the fourth and the last ultrasonographic examination. At the second measurement, the ventral prostate area of animals from $\mathrm{PCa}$ group was lower when compared with the control group $(p<0.05)$. The opposite was observed at the fourth $(p<0.05)$ (Figure 1, upper panel). Although the difference did not reach statistical significance, the dorsal prostate area increased between fourth and fifth measurement (Figure 2, upper panel).

The echogenicity of the ventral prostate lobes increased throughout the study. In the last ultrasonographic examination, hypoechoic or anechoic round to oval structures were observed in the ventral and dorsal prostate of animals from the control group $(n=3)$ and PCa group $(n=3)$ (Figures 1 and 2, lower panels). A poor contrast enhancement was observed for both groups.

Prostate macroscopic and microscopic analysis. At sacrifice, macroscopic evaluation of the prostate revealed slight enlargement and irregular surface. Moreover, three animals from the PCa group presented small yellowish nodular lesions (less than $1 \mathrm{~mm}$ ) in the dorsolateral prostate lobe (Figure 2, lower panel).

Histopathological findings for prostatic lesions are summarized in Table IV. The ventral prostate showed normal 


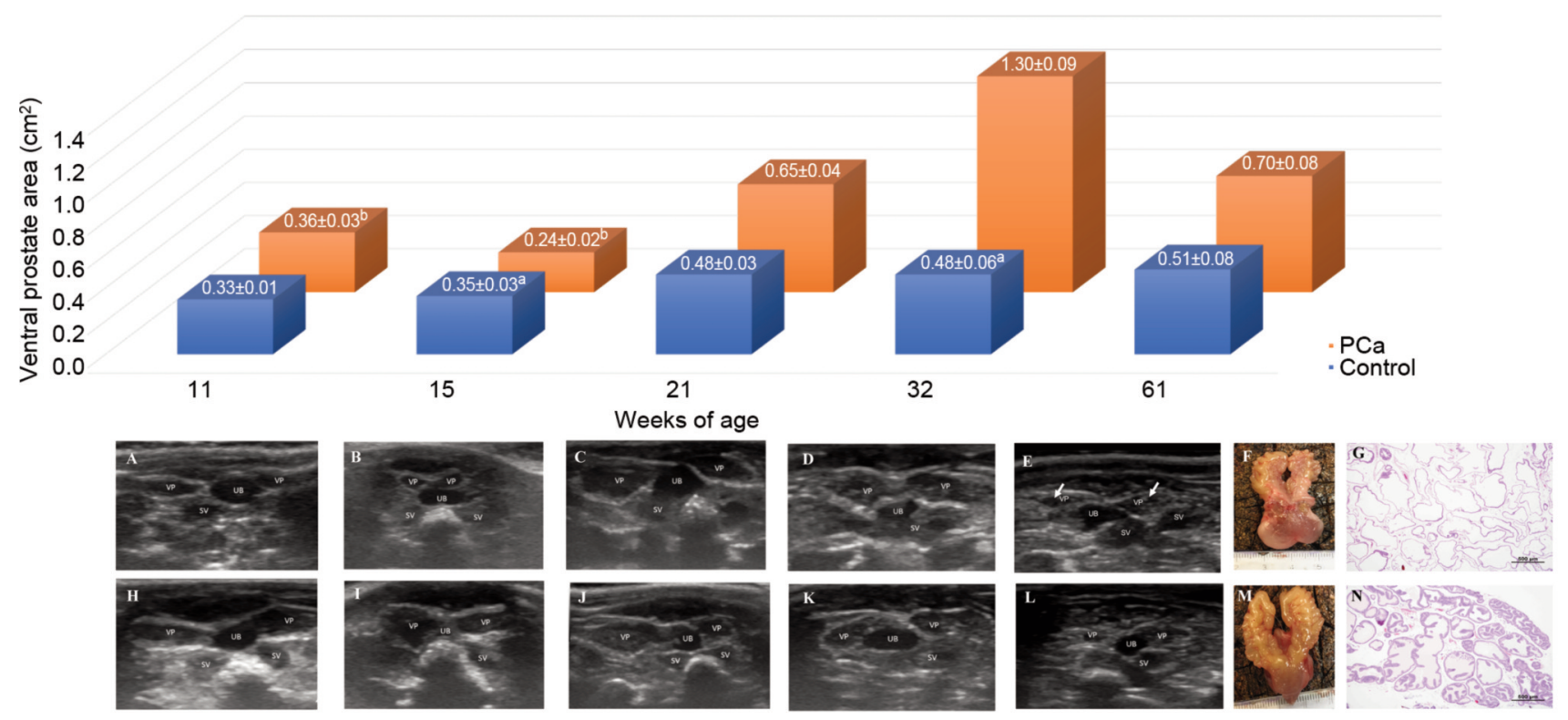

Figure 1. Ultrasonographic monitoring of rat prostate during the experimental protocol in prostate cancer (PCa) and control groups. The chart (upper panel) shows the ventral prostate area by ultrasonography at 11,15,21, 32 and 61 weeks of age. The lower panel shows images from ultrasonographic monitoring of rat prostate in the PCa $(A-E)$ and control $(H-L)$ groups at corresponding ages as well as gross and histological views of resected prostate $(F, G$ and $M, N$, respectively). The ventral prostate lobes $(V P)$ and seminal vesicles $(S V)$ were observed surrounding the urinary bladder $(U B)$. A variation of the prostate lobes area and echogenicity was observed during the experiment. In the last examination, ventral prostate of PCa animals exhibited hypoechoic or anechoic round to oval structures (arrows) (E), compatible with the nodular lesions observed at prostate macroscopic evaluation after animals' sacrifice $(F)$ and the lesions observed in the microscopic analysis. Ventral prostate lobes of PCa animals showed atrophic acini coexisting with occasional normal acini $(G)$. Ventral prostate of normal animals presented small concretions $(N)$. ${ }^{a}$ Statistically different from PCa group $(p<0.05) ;{ }^{b}$ statistically different from weeks 21,32 and $61(p<0.05)$. Bars $=500 \mu \mathrm{m}$.

acini coexisting with minimal to mild areas of atrophic acini, lined by non-atypical, low cuboidal to flat cells, with absence or small epithelial folds, and occasional cell vacuoles. Although it was observed in both groups, atrophy was more extensive in the PCa group. Most rats from the PCa group (13/14) showed mild to moderate atrophy, whereas only one out of the eight control animals developed mild atrophy $(p<0.05)$. Surrounding the atrophic acini, there was an increased quantity of stroma infiltrated by mast cells and occasionally mononucleated cells, and small dilated vessels. Multiple concretions (i.e. corpora amylacea), frequently calcified, were observed inside the gland lumen in both experimental groups. Inflammation was rarely observed in this prostate lobe.

The dorsal prostate showed minimum to mild peripheral papillary hyperplasia in both groups, and lobes were filled with pink flocculent secretory material. However, lesions were evaluated in association with those observed in lateral prostate.

The dorsolateral prostate from both groups showed acute serous inflammation with and without purulent acini inflammation, however, it was more extensive in the $\mathrm{PCa}$ group. Areas of acinar inflammation showed epithelial reactive hyperplasia with cell stratification, and light cellular and nuclear atypia. Focal chronic interstitial inflammation with focal stromal fibrosis was also observed in both groups. As expected, a higher number of pre-neoplastic and neoplastic lesions in the dorsolateral prostate was observed in the PCa group (14/14), when compared to controls animals $(5 / 8)(p<0.05)$. Most rats of the PCa group presented multiple pre-neoplastic and neoplastic lesions (12/14; $85.7 \%$ ), but no animal from the control group showed multiple lesions. Only one out of the eight animals from the control group developed carcinoma, whereas 12/14 animals from the PCa group developed neoplasia.

\section{Discussion}

The prostate is the largest accessory gland of the male reproductive tract (1). Interestingly in contrast to what is believed by the majority of people, the prostate is not exclusive to males and may be found in the reproductive tract of female Mongolian gerbils (12). PCa is one of the leading causes of cancer-related deaths in developing countries, being a disease of increasing medical significance around the world and the focus of intense investigation (4). $\mathrm{PCa}$ is considered a heterogeneous disease, ranging from 


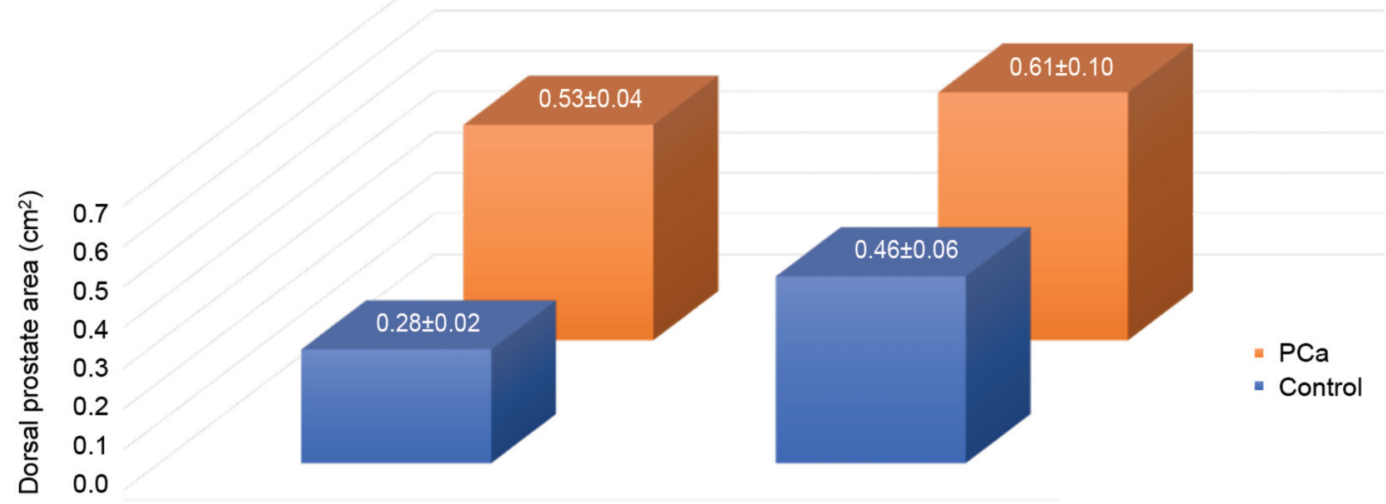

32
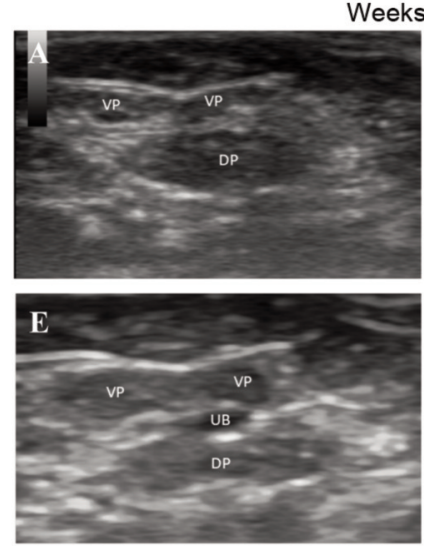

Weeks of age
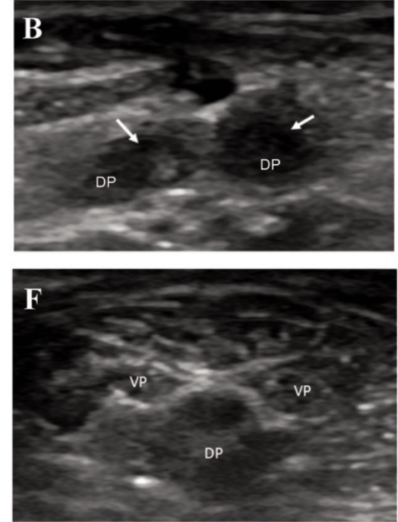
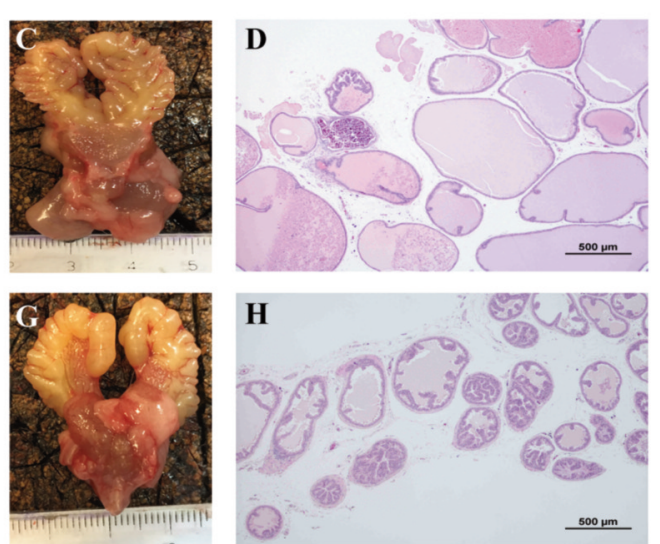

Figure 2. Ultrasonographic monitoring of rat prostate during the experimental protocol in prostate cancer (PCa) and control groups. The chart (upper panel) shows the dorsal prostate area in both groups as measured by ultrasonography at 32 and 61 weeks of age. The lower panel shows Images from ultrasonographic monitoring of rat prostate in the $P C a(A$ and $B)$ and control (E and $F)$ groups at corresponding ages as well as gross and histological views of resected prostate $(C, D$ and $G, H$, respectively). The dorsal prostate $(D P)$ was observed near the urinary bladder (UB). $A$ variation of the dorsal prostate area was observed between the examinations. In the last examination, dorsal prostate of PCa animals exhibited hypoechoic or anechoic round to oval structures (arrows) (B), compatible with the nodular lesions observed at prostate macroscopic evaluation after animal sacrifice $(C)$ and the lesions observed in the microscopic analysis $(D)$. Dorsolateral prostate lobes of PCa animals presented acini dilation and inflammation $(D)$. Dorsal prostate of normal animals showed normal and hyperplastic acini $(H)$. Bars $=500 \mu \mathrm{m}$.

asymptomatic disease to a quickly progressive systemic malignancy (13). Although several species are available as models to study different diseases, for $\mathrm{PCa}$, the rat is the most frequently used (7-9). Indeed, rats are relatively cheap, they are small animals, easy to accommodate and manipulate, their biology and physiology are well known, and most importantly, they are mammals. The chemically and hormonally induced model used in this experiment is also one of the most frequently chosen ones for the study of prostate carcinogenesis (7-9).

The variation of body weight between control and cancerinduced animals is an important tool for assessing an animal's health status during experimental assays. The mean animal body weight was lower in the PCa group when compared with the control group due to carcinogenesis.
Weight loss is frequently observed in several types of cancer, being associated with reduced survival and poorer quality of life in patients (14). The prostate weight was higher in PCa groups due to the development of multiple non-neoplastic and neoplastic lesions $(p<0.05)$.

Despite the frequent use of the rat model of chemically and hormonally induced PCa, follow-up study using a non-invasive imaging tool to monitor the prostate changes during the process of cancer induction has never been reported to our knowledge. Imaging is considered a powerful tool because most imaging techniques are non or minimally invasive, provide dynamic real-time data and repeated measurements (13). In humans, ultrasonography may not allow the detection of early disease and only provides limited information on disease staging (1518). Nevertheless, transrectal ultrasonography is frequently 
Table IV. Histological lesions of ventral and dorsal prostate lobes from control and prostate cancer ( $\mathrm{PCa}$ ) groups.

\begin{tabular}{llcc}
\hline Lesions & & \multicolumn{2}{c}{ Group } \\
\cline { 3 - 4 } & & Control (n=8) & PCa (n=14) \\
\hline Ventral prostate & $<10 \%$ & 7 & 1 \\
Atrophy & $10-39 \%$ & 1 & $9^{\mathrm{a}}$ \\
& $40-50$ & 0 & 4 \\
Concretions & & 8 & 14 \\
Dorsolateral prostate & & & \\
Acute inflammation & $<10 \%$ & 2 & 0 \\
& $10-39 \%$ & 8 & 0 \\
& $40-50 \%$ & 0 & 7 \\
Chronic interstitial & $>50 \%$ & 0 & 7 \\
inflammation & $10-39 \%$ & 8 & 14 \\
Preneoplastic lesions & Overall & 4 & $13^{\mathrm{a}}$ \\
Neoplastic lesions & Overall & 1 & $9^{\mathrm{a}}$ \\
& Total & 39 & $78^{\mathrm{a}}$ \\
\hline
\end{tabular}

aSignificantly different from the control group $(p<0.05)$. No other statistically significant differences were found between groups/lesions.

used to estimate prostate volume and to direct the biopsy needle to a desired anatomical location (13). The evaluation of rat prostate at intermediate timepoints, although more crucial, provides continuous information on carcinogenesis. In this study, ultrasonography was performed before (11 weeks of age) beginning the multistep protocol for PCa induction, after flutamide administration (15 weeks of age), and 6,20 and 46 weeks after MNU administration (i.e. at 21,35 and 61 weeks of age).

Ventral and dorsal prostate lobes were observed encircling the urinary bladder. Ventral prostate lobes were observed in all ultrasonographic examinations, with an increase of echogenicity throughout the study. This was probably associated with atrophy and the presence of solid, sometimes calcified, concretions in the gland lumen. The dorsal prostate lobe was only observed in the last two examinations, probably due to its low echogenicity or small size, which hampered its visualization at the earlier timepoints. Its observation at the last two timepoints was possibly due to age-related hyperplasia.

The area of ventral prostate of animals from the control group increased with animal growth. The area of ventral prostate lobes from the PCa group decreased between the first and the second examinations due to flutamide administration. Flutamide is a nonsteroidal anti-androgen that acts by inhibiting the binding of dihydrotestosterone to the target cell receptor, interfering with androgen action (19). A single administration of testosterone propionate and the carcinogen agent MNU promoted prostate growth in rats of the PCa group, as reflected by the increase of prostatatic area at 21 and
32 weeks of age. This increase in prostatic dimensions may be related to the proliferative effect of androgens on prostate tissue, as previously described by Limonta et al. (20). As silastic tubes filled with crystalline testosterone were subcutaneously implanted into the interscapular region, a continuous increase or at least maintenance of the prostate area registered at 32 weeks of age was expected. However, a reduction of ventral prostate area was observed at 61 weeks of age. Although the reason for the reduction of the ventral prostate lobes is not completely clear, it may be associated with a reduction of testosterone release rate by the implants approximately 5.8 months after implantation (21). In previous work, Sato et al. (22), replaced testosterone implants at 6week intervals.

Ventral prostate atrophy was observed histologically, corroborating the ultrasonographic findings. Prostatic atrophy may be related to androgen decline with aging, as previously described by Lau et al. (23) in rats aged more than 9 months; the animals from our study were 15 months old at sacrifice. Although diffuse prostate atrophy is usually associated with a decrease in circulating androgens, in contrast, focal atrophy is not (24). Concretions are a very common age-related change and are generally associated with acinar atrophy (25).

The dorsal prostate area increased in all groups between the fourth and the fifth ultrasonographic examinations, accompanying animal growth. The poor contrast enhancement was probably due to the low vascularization of the prostate itself, low vessel caliber or small prostatatic lesions, as observed microscopically. Ventral and dorsal prostate lobes were visible in ultrasonographic examinations. Ultrasonography allowed a comprehensive and detailed study of the rat prostate, with the detection of the variation of prostate size due to the protocol of $\mathrm{PCa}$ induction or animal growth. Moreover, it allowed the detection of small hypoechoic or anechoic round to oval structures compatible with the lesions identified in the histological analysis of the prostate. In this way, it is a non-invasive tool recommended for PCa monitoring.

\section{Conflicts of Interest}

None declared.

\section{Authors' Contributions}

Ana I. Faustino-Rocha collaborated in the cancer induction, performed the ultrasound evaluation and wrote the article. Fernanda Seixas performed the histological analysis of the prostate and supervised the article writing. Rita Ferreira and Margarida Fardilha designed the experimental protocol, and supervised the work and article writing. Jessica Silva and Maria J. Pires collaborated in the experimental protocol. Mário Ginja and Paula A. Oliveira designed the experiment, participated in the execution and supervision of the experimental work, and supervised the article writing. 


\section{Acknowledgements}

This work was supported by European Investment Funds by FEDER/COMPETE/POCI - Operational Competitiveness and Internationalization Program, and National Funds by FCT Portuguese Foundation for Science and Technology, under the project UIDB/04033/2020 and the project RUNawayPCa (POCI-010145-FEDER-006958 and PTDC/DTP-DES/6077/2014).

\section{References}

1 Lee $\mathrm{CH}$, Akin-Olugbade $\mathrm{O}$ and Kirschenbaum A: Overview of prostate anatomy, histology, and pathology. Endocrinol Metab Clin North Am 40: 565-575, 2011. PMID: 21889721. DOI: 10.1016/j.ec1.2011.05.012

2 Bhavsar A and Verma S: Anatomic imaging of the prostate. Biomed Res Int 2014: 1-9, 2014. PMID: 25243174. DOI: 10.1155/ 2014/728539

3 Johnston SD, Kamolpatana K, Root-Kustritz M V and Johnston GR: Prostatic disorders in the dog. Anim Reprod Sci 60-61: 405-415, 2000. PMID: 10844211. DOI: 10.1016/s0378-4320(00)00101-9.

4 International Agency for Research on Cancer - World Health Organization. Incidence, Mortality and Prevalence by Cancer Site. Globocan 2018, 1-2, 2019.

5 Pietro G Di, Chornokur G, Kumar NB, Davis C and Park JY: Racial differences in the diagnosis and treatment of prostate cancer. Int Neurourol J 20: S112-119, 2016. PMID: 27915474. DOI: $10.5213 /$ inj.1632722.361

6 Discacciati A and Wolk A: Lifestyle and dietary factors in prostate cancer prevention. Recent Results Cancer Res 202: 2737, 2014. PMID: 24531774. DOI: 10.1007/978-3-642-45195-9_3

7 Nascimento-Gonçalves E, Faustino-Rocha AI, Seixas F, Ginja M, Colaço B, Ferreira R, Fardilha M and Oliveira PA: Modelling human prostate cancer: Rat models. Life Sci 203: 210-224, 2018. PMID: 29684445. DOI: 10.1016/j.lfs.2018.04.014

8 Shirai T: Significance of chemoprevention for prostate cancer development: experimental in vivo approaches to chemoprevention. Pathol Int 58: 1-16, 2007. PMID: 18067635. DOI: $10.1111 / \mathrm{j} .1440-1827.2007 .02182 . x$

9 Shirai T, Takahashi S, Cui L, Futakuchi M, Kato K, Tamano S and Imaida K: Experimental prostate carcinogenesis - rodent models. Mutat Res 462: 219-226, 2000. PMID: 10767633. DOI: $10.1016 / \mathrm{s} 1383-5742(00) 00039-9$

10 Forbes D, Blom H, Kostomitsopulos N, Moore G and Perretta G: Euroguide On the Accommodation and Care of Animals Used for Experimental and Other Scientific Purposes. London, Federation of European Laboratory Animal Science Associations, 2007. Available at: http://www.felasa.eu/ ?ACT=43\&file_id=YvnrKyYgFg7JnMiaTyWer1Qucb6z3BI4Cg p2kc4xohQ\%3D\&access=koed0wb4RMzvR\%2BLcdXr0sEi1Oq BKK82A7S2pXszotEQ\%3D

11 Bosland MC: Animal models for the study of prostate carcinogenesis. J Cell Biochem Suppl 16H: 89-98, 1992. PMID: 1289679. DOI: $10.1002 /$ jcb.240501221

12 Custodio AMG, Santos FCA, Campos SGP, Vilamaior PSL, Góes RM and Taboga SR: Aging effects on the Mongolian gerbil female prostate (Skene's paraurethral glands): Structural, ultrastructural, quantitative, and hormonal evaluations. Anat Rec Adv Integr Anat Evol Biol 291: 463-474, 2008. PMID: 18231985. DOI: $10.1002 /$ ar.20637
13 Kelloff GJ, Choyke P, Coffey DS and Prostate Cancer Imaging Working Group: Challenges in clinical prostate cancer: Role of imaging. Am J Roentgenol 192: 1455-1470, 2009. PMID: 19457806. DOI: 10.2214/AJR.09.2579

14 Strasser F and Bruera ED: Update on anorexia and cachexia. Hematol Oncol Clin North Am 16: 589-617, 2002. PMID: 12170570. DOI: 10.1016/s0889-8588(02)00011-4

15 DeGrado TR, Coleman RE, Wang S, Baldwin SW, Orr MD, Robertson CN, Polascik TJ and Price DT: Synthesis and evaluation of $18 \mathrm{~F}$-labeled choline as an oncologic tracer for positron emission tomography: initial findings in prostate cancer. Cancer Res 61: 110-117, 2001. PMID: 11196147.

16 Price DT, Coleman RE, Liao RP, Robertson CN, Polascik TJ and DeGrado TR: Comparison of [18 F]fluorocholine and [18 F]fluorodeoxyglucose for positron emission tomography of androgen dependent and androgen independent prostate cancer. J Urol 168: 273-280, 2002. PMID: 12050555.

17 Kwee SA, Wei H, Sesterhenn I, Yun D and Coel MN: Localization of primary prostate cancer with dual-phase $18 \mathrm{~F}$-fluorocholine PET. J Nucl Med 47: 262-269, 2006. PMID: 16455632.

18 Albrecht S, Buchegger F, Soloviev D, Zaidi H, Vees H, Khan HG, Keller A, Bischof Delaloye A, Ratib O and Miralbell R: ${ }^{11} \mathrm{C}$-Acetate PET in the early evaluation of prostate cancer recurrence. Eur J Nucl Med Mol Imaging 34: 185-196, 2007. PMID: 16832632. DOI: 10.1007/s00259-006-0163-x

19 Goldspiel BR and Kohler DR: Flutamide: An antiandrogen for advanced prostate cancer. Ann Pharmacother 24: 616-623, 1990. PMID: 2193461. DOI: 10.1177/106002809002400612

20 Limonta P, Dondi D, Marelli MM, Moretti RM, Negri-Cesi P and Motta M: Growth of the androgen-dependent tumor of the prostate: Role of androgens and of locally expressed growth modulatory factors. J Steroid Biochem Mol Biol 53: 401-405, 1995. PMID: 7626487. DOI: 10.1016/0960-0760(95)00086-f

21 Kelleher S, Howe C, Conway AJ and Handelsman DJ: Testosterone release rate and duration of action of testosterone pellet implants. Clin Endocrinol 60: 420-428, 2004. PMID: 15049955. DOI: 10.1111/j.1365-2265.2004.01994.x

22 Sato S, Suzuki S, Naiki-Ito A, Komiya M, Ne L, Kato H, Sagawa H, Yamashita Y, Shirai $T$ and Takahashi S: Establishment of an invasive prostate cancer model in transgenic rats by intermittent testosterone administration. J Toxicol Pathol 27: 43-49, 2014. PMID: 24791066. DOI: 10.1293/tox.2013-0052

23 Lau KM, Tam NNC, Thompson C, Cheng RYS, Leung YK and Ho SM: Age-associated changes in histology and gene-expression profile in the rat ventral prostate. Lab Investig 83: 743-757, 2003. PMID: 12746483. DOI: 10.1097/01.lab.0000069519.06988.24

24 De Marzo AM, Marchi VL, Epstein JI and Nelson WG: Proliferative inflammatory atrophy of the prostate Implicationstions for prostatic carcinogenesis. Am J Pathol 155: 1985-1992, 1999. PMID: 10595928. DOI: 10.1016/S00029440(10)65517-4

25 Creasy D, Bube A, de Rijk E, Kandori H, Kuwahara M, Masson R, Nolte T, Reams R, Regan K, Rehm S, Rogerson P and Whitney K: Proliferative and nonproliferative lesions of the rat and mouse male reproductive system. Toxicol Pathol 40, 2012. PMID: 22949412. DOI: 10.1177/0192623312454337

Received April 5, 2020

Revised April 15, 2020

Accepted April 17, 2020 